\title{
Pulsational velocity fields in the atmospheres of two roAp stars HR 1217 and $\gamma$ Equ
}

\author{
M. Sachkov ${ }^{1}$, T. Ryabchikova ${ }^{1,2}$, O. Kochukhov ${ }^{3}$, W.W. Weiss ${ }^{2}$, \\ P. Reegen ${ }^{2}$, J.D. Landstreet ${ }^{4}$ \\ ${ }^{1}$ Institute of Astronomy, Russian Academy of Science, Pyatnitskaya 48 , \\ 119017 Moscow, Russia \\ ${ }^{2}$ Institute for Astronomy, University of Vienna, Türkenschanzstraße 17, \\ 1180 Vienna, Austria \\ ${ }^{3}$ Uppsala Astronomical Observatory, Box 515, 75120 Uppsala, Sweden \\ ${ }^{4}$ Department of Astronomy, University of Western Ontario, London, \\ Ontario N6A 3K7, Canada
}

\begin{abstract}
We discuss spectroscopic observations of pulsations in the roAp stars $\gamma$ Equ and HR 1217. In both stars we observe strong variations of rare-earth lines and detect running magneto-acoustic waves, but do not find evidence for a standing pulsational wave. In $\gamma$ Equ we find strong pulsational variations across line profiles, characteristic of $\ell \geq 2$ modes.
\end{abstract}

\section{Observations and radial velocity measurements}

We observed $\gamma$ Equ and HR 1217 using the single-order $f / 4$ Gecko coudé spectrograph at the $3.6-\mathrm{m}$ Canada-France-Hawaii telescope with $R \approx 115000$ during 2001 October 2-4 and 2002 September 22-25. The exposure time for HR 1217 was $60 \mathrm{~s}(\mathrm{~S} / \mathrm{N} \approx 100)$ and for $\gamma$ Equ was $90 \mathrm{~s}(\mathrm{~S} / \mathrm{N} \approx 150)$. Our observations covered 7 spectral regions centered at $4860,5300,5855,6160,6600,6675$ and $7780 \AA$ with the most important spectral lines: singly and doubly-ionized REE, $\mathrm{H} \alpha, \mathrm{H} \beta$, $\mathrm{O}$ I, Fe I, Ca I and Ba II. Observations of HR 1217 were obtained at 7 rotational phases in the interval $0.24-0.83$ with the zero point near magnetic maximum. High S/N and spectral resolution of the observations allow us to carry out an analysis of radial velocity (RV) variations across individual spectral lines using two kinds of measurements: moment (center-of-gravity) and bisector. Fig. 1 shows RV variations for a set of $\mathrm{Nd}$ lines of different ionisation stages and different intensities in spectrum of $\gamma$ Equ. This plot clearly demonstrates how large RV variations across the spectral line may be. Note, that both kinds of measurements produce similar results up to residual intensity 0.95 , where most of the lines are measured. Fig. 1 also demonstrates how careful one should be using a moment technique. There are no absolutely unblended lines, therefore the area of integration is usually cut at some level below the continuum. However, the value of RV in stars like $\gamma$ Equ strongly depends on the measurement cut-off. This may lead to a spurious discrepancy between RV amplitudes of different spectral lines. 


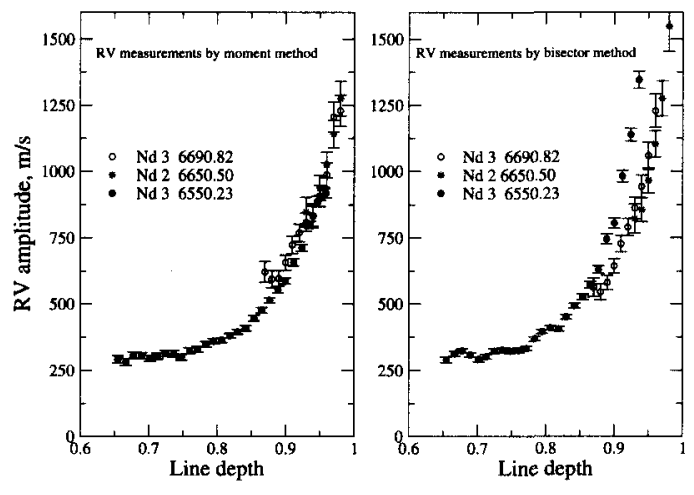

Figure 1. RV measurements by the moment method (left panel) and the bisector method (right panel) across spectral line profiles in $\gamma$ Equ.

All RV measurements in $\gamma$ Equ, which has extremely sharp and strong lines, were made by bisectors, while in HR 1217 , with its weaker and wider spectral lines $\left(v \sin i=5.6 \mathrm{~km} \mathrm{~s}^{-1}\right)$, we carried out RV measurements with the moment method. Amplitudes and phases of the pulsational RV curves were derived with non-linear least-squares fitting.

Two main photometric frequencies at $2.721 \mathrm{mHz}$ and $2.687 \mathrm{mHz}$ were found from our spectroscopic data for HR 1217. Other frequencies were not possible to obtain independently due to the scarcity of the observations. We observed both rotational modulation and the beating effect between close frequencies. The last effect produces extra difficulties in RV pulsational studies. For $\gamma$ Equ we found a large change in RV amplitudes between the $1999\left(\approx 800 \mathrm{~m} \mathrm{~s}^{-1}\right), 2001$ $\left(\approx 1200 \mathrm{~m} \mathrm{~s}^{-1}\right)$ and $2002\left(\approx 500 \mathrm{~m} \mathrm{~s}^{-1}\right)$ observing seasons. This is not a rotational modulation because of the extremely long rotational period of the star $(77 \mathrm{yr})$.

\section{RV line analysis and mode identification}

RV of more than 40 spectral lines were measured in the spectra of each star. These lines are: O I (IR triplet), Fe I, Ca I, Ba II, Ce II, Pr II, Pr III, Nd II, Nd III, Sm II, Eu IIand Tb III. The lines of O I, Fe I, Ca Iand Ba II do not show pulsational variations above the error of the amplitude measurements which varies between 10 and $50 \mathrm{~m} \mathrm{~s}^{-1}$, depending on the line strength. All lines of the rare-earth elements show significant amplitudes with a different behaviour across individual spectral lines in $\gamma$ Equ and HR 1217 (Fig. 2). In $\gamma$ Equ variations of RV with line depth are opposite in $\mathrm{H} \alpha$ and in REE lines. Radial velocities across the $\mathrm{H} \alpha$ core decrease from the center to the base of the core, as was observed for two other roAp stars, $\alpha$ Cir (Baldry et al. 1999) and HR 3831 (Baldry \& Bedding 2000).

We performed single mode theoretical pulsational calculations for a typical line for different inclinations of the pulsational axis and different combinations of $\ell$ and $m: \ell=1,2,3, m \leq \ell$. RV amplitude dependence on the line depth in 

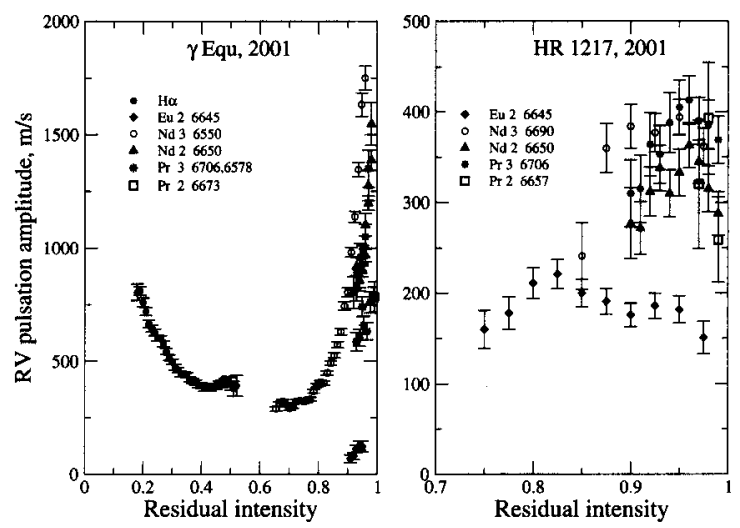

Figure 2. RV amplitudes across spectral line profiles in $\gamma$ Equ (left panel) and in HR 1217 (right panel).

HR 1217 is well reproduced by dipole mode. RV amplitude dependence on the line depth in $\gamma$ Equ requires modes with $\ell \geq 2$, which confirms mode identification proposed by Kochukhov \& Ryabchikova (2001). For both stars variations of the second moment $\left\langle V^{2}\right\rangle$ cannot be explained by any single mode.

\section{Running versus standing waves in the atmosphere of roAp stars}

Recently Kurtz, Elkin \& Mathys (2003) performed similar pulsational analysis of another roAp star, HD 166473, and claimed to find a separation between regions of standing and running waves in the atmosphere, e.g., a magneto-acoustic reflectory boundary region. Their suggestion was based on the analysis of phase shifts. They did not find any phase shift between pulsational curves for different depths in the $\mathrm{H} \alpha$ core (an indication of the standing wave), while they found a clear phase difference for Pr II and Pr III lines of different intensity (an indication of running wave). For $\gamma$ Equ a plot of residual intensities versus pulsation phase (Fig. 3), similar to that in Kurtz et al., indicates no standing wave region in the atmosphere: phases of pulsation maximum show variations for both the $\mathrm{H} \alpha$ core and for individual REE spectral lines. The same conclusion is valid for HR 1217. Why did Kurtz et al. find a standing wave? There may be two reasons. First, phase error in their analysis is \pm 0.05 of the pulsation period (vertical dashed lines in our plots). One immediately sees that phase variations in the core of $\mathrm{H} \alpha$ line lie inside these error limits. Second, one can say that different kinds of pulsation waves may exist in different roAp stars. In any case, the detection of a magneto-acoustic reflectory boundary layer needs confirmation. 


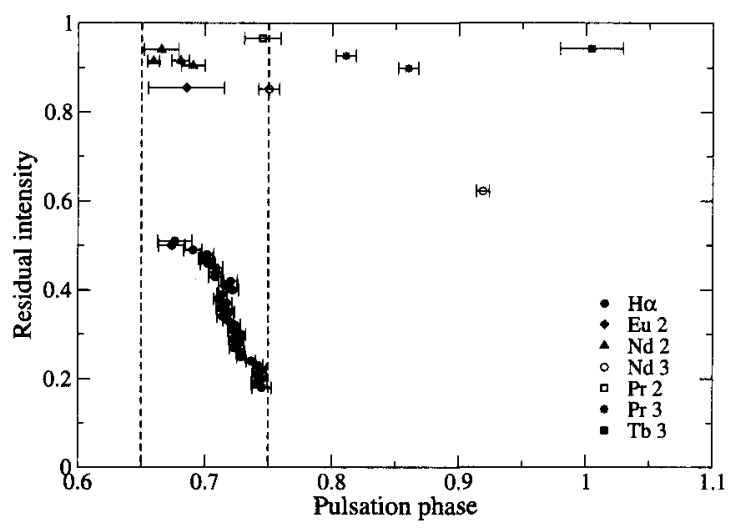

Figure 3. Phase of RV pulsation maximum for the $\mathrm{H} \alpha$ core at different depths and for spectral lines of different elements and ions in $\gamma$ Equ.

\section{Pulsation and element stratification}

Ryabchikova et al. (2002) performed empirical stratification analysis of the atmosphere of $\gamma$ Equ and found that they need concentration of $\mathrm{Pr}$ and $\mathrm{Nd}$ in the upper atmospheric layers to explain the intensities of $\mathrm{Pr}$ and $\mathrm{Nd}$ lines of the first and second ionization stages. We performed a similar stratification analysis for $\mathrm{Pr}, \mathrm{Nd}$ and $\mathrm{Tb}$ in the atmosphere of HR 1217 . Using empirical abundance stratification we calculated formation depths of REE lines and points in the $\mathrm{H} \alpha$ core. A plot of RV amplitudes versus optical depths provides independent evidence for the pulsating waves in the atmosphere of roAp stars, propagating from the photospheric layers to the outer atmosphere with the increasing amplitudes. These results give important constraints for the theory of stellar pulsations governed by magnetic fields.

Acknowledgments. This work was supported by the Fonds zur Förderung der wiss. Forschung (project P14984), by the Jubiläumsfonds der Österr. Nationalbank (project No. 7650), by the Natural Sciences and Engineering Research Council of Canada, by RFBR (grants 02-02-16677, 03-02-26791), and by Russian Federal program "Astronomy" (part 1102).

\section{References}

Baldry, I.K., Bedding, T.R. 2000, MNRAS, 318, 341

Baldry, I.K., Viskum, M., Bedding, T.R., et al. 1999, MNRAS, 302, 381

Kochukhov, O., Ryabchikova, T. 2001, A\&A, 374, 615

Kurtz, D.W., Elkin V.G., Mathys, G. 2003, MNRAS, 343, L5

Ryabchikova, T., Piskunov, N., Kochukhov, O., et al. 2002, A\&A, 384, 545 